\title{
Comparison of Efficiency Between Corticosteroid and Platelet Rich Plasma Injection Therapies in Patients With Knee Osteoarthritis
}

\author{
Ece USLU GÜVENDİ®, ${ }^{1}$ Ayhan AŞKIN@,${ }^{2}$ Güven GÜVENDİi, ${ }^{3}$ Hikmet KOÇYİĞİT (i) ${ }^{2}$ \\ ${ }^{1}$ Department of Physical Medicine and Rehabilitation, Dumlupınar University \\ Evliya Çelebi Training and Research Hospital, Kütahya, Turkey \\ ${ }^{2}$ Department of Physical Medicine and Rehabilitation, Katip Çelebi University \\ Atatürk Training and Research Hospital, İzmir, Turkey \\ ${ }^{3}$ Department of Physiology, Dokuz Eylül University Faculty of Medicine, İzmir, Turkey
}

\begin{abstract}
Objectives: This study aims to assess whether platelet rich plasma (PRP) is an effective treatment for knee osteoarthritis (OA), and compare its efficiency with corticosteroid treatment in terms of pain control, physical function, and quality of life.

Patients and methods: The study included 50 patients (4 males, 46 females; mean age $61.6 \pm 6.9$ years; range 50 to 75 years) who were diagnosed as grade 3 knee OA. Patients were randomized into three groups as corticosteroid group (receiving one corticosteroid injection), single PRP group (receiving one PRP injection), and three PRP group (receiving three PRP injections with one week interval). All patients were given a home exercise program. Patients were evaluated with Visual Numeric Scale (VNS), Western Ontario and McMaster Universities Arthritis Osteoarthritis Index (WOMAC), Lequesne index and the Hospital Anxiety and Depression Scale before treatment, and at second and sixth months following the implementation of injections.

Results: In single and three PRP groups; VNS, WOMAC and Lequesne scores decreased significantly at second month follow-up; also, sixth month scores showed a slight increase but remained significantly lower than baseline. In corticosteroid group, all VNS, WOMAC, and Lequesne scores decreased at second month follow-up; however, at sixth month, rest, night VNS and WOMAC stiffness scores were increased while no significant difference was found with baseline. At sixth month; VNS movement, WOMAC pain, function, and the total and Lequesne scores were worse, but remained significantly lower than baseline. When groups were compared, three PRP group's second month VNS movement scores were significantly lower than the corticosteroid group. Sixth month VNS movement and WOMAC pain scores were significantly lower in single and three PRP groups compared to the corticosteroid group, with no significant difference between the PRP groups.

Conclusion: Our findings revealed that PRP is a safe treatment option and efficient in OA symptom control up to six months after application. Treatment response obtained with corticosteroid injection has a shorter duration than PRP treatment.

Keywords: Corticosteroid; intraarticular injection; knee osteoarthritis; platelet rich plasma.
\end{abstract}

Osteoarthritis (OA) is a slowly progressive, chronic degenerative disease that is characterized with varying degrees of joint cartilage loss with local inflammation and periarticular bone rebuild. ${ }^{1}$ The progression of cartilage lesions manifests with pain, stiffness, swelling, decreased joint range of motion while significantly affecting the quality of life. Treatment is focused on reducing symptoms and slowing the progression of the disease. It includes physical therapy modalities, orthoses, pharmacological treatments, and surgical interventions. Patients resistant to topical and oral pharmacological treatments can benefit from intraarticular injections. ${ }^{2}$

Corticosteroid and hyaluronic acid injections are the most commonly used agents for intraarticular treatment. Intraarticular steroid injections in knee $\mathrm{OA}$ are also among the recommendations of

Received: August 05, 2017 Accepted: October 05, 2017 Published online: November 02, 2017

Correspondence: Ayhan Aşkın, MD. Katip Çelebi Üniversitesi, Atatürk Eğitim ve Araştırma Hastanesi, Fiziksel Tıp ve Rehabilitasyon Anabilim Dalı, 35360 Karabağlar, İzmir, Turkey. Tel: +90 232 - 2444444 e-mail: ayhanaskin@gmail.com

\section{Citation:}

Uslu Güvendi E, Aşkın A, Güvendi G, Koçyiğit H. Comparison of Efficiency Between Corticosteroid and Platelet Rich Plasma Injection Therapies in Patients With Knee Osteoarthritis. Arch Rheumatol 2018;33(3):273-281. 
"Osteoarthritis Research Society International 2014" and "American College of Rheumatology (ACR) 2012" guidelines. ${ }^{3,4}$ The disadvantage of corticosteroid injections is its short duration of benefit. ${ }^{5}$ Another commonly used treatment in knee OA is synthetic hyaluronic acid due to its modulating effects on inflammatory reactions and viscosupplementation. Hyaluronic acid's natural form can be found in healthy joint fluid and studies that demonstrate superiority over corticosteroid injections are available; however, an up-to-date meta-analysis has emphasized clinical ineffectiveness and increased risk of serious side effects. ${ }^{6}$

The ability of the damaged cartilage to heal is insufficient due its isolation of systemic circulation. Treatment modalities are focused to provide normal tissue homeostasis, restore and slow down the resulting structural damage and to delay the need for invasive surgery as much as possible. The role of complex regulation of growth factors is important to protect normal tissue structure and repair the tissue damage. In last few years, growth factor applications to damaged tissues have become a popular treatment option. Platelet rich plasma (PRP) contains four-five times more platelet concentration than normal blood and has more intense amounts of growth factor. It is a simple, low-cost and minimally invasive method for obtaining autologous growth factors. ${ }^{7}$ There are many case series in the literature that show positive results regarding intraarticular PRP injections. However, relatively few randomized controlled trials are available. Most often, these trials have been conducted to compare shortterm clinical outcomes with hyaluronic acid. In conclusion, PRP has been reported as a welltolerated, appropriate treatment option in early stage knee OA. ${ }^{8}$

There are studies in the literature that include PRP-PRP, PRP-placebo, and PRP-hyaluronic acid comparisons; however, studies comparing corticosteroid-PRP injections in knee OA are significantly fewer. ${ }^{9}$

Therefore, in this study, we aimed to assess whether PRP is an effective treatment for knee $\mathrm{OA}$, and compare its efficiency with corticosteroid treatment in terms of pain control, physical function, and quality of life.

\section{PATIENTS AND METHODS}

This single-center, prospective, randomized, single-blind study was conducted at Katip Çelebi University Atatürk Training and Research Hospital between June 2015 and March 2016 and included 57 patients ( 7 males, 50 females; mean age $61.3 \pm 6.7$ years; range 50 to 75 years) diagnosed with primary knee $\mathrm{OA}$ according to ACR criteria and classified with Kellgren-Lawrence classification scale as grade $3 \mathrm{OA}$. Seven patients were lost to follow-up (Figure 1) and the study was completed with 50 patients (4 males, 46 females; mean age $61.6 \pm 6.9$ years; range 50 to 75 years). Exclusion criteria included diagnosis of secondary OA, usage of more than three months of corticosteroids or drugs that inhibit platelet aggregation (non-steroidal antiinflammatory drugs, acetyl salicylic acid, thienopyridines, cyclopentyltriazolo-pyrimidines, glycoprotein IIB-IIla complex inhibitors, phosphodiesterase inhibitors) in last seven days, skin lesions on the knee joint, presence of anemia or thrombocytopenia (hemoglobin $<12 \mathrm{gr} / \mathrm{dL}$, platelet $<150000 \mathrm{~K} / \mu \mathrm{L}$ ), diagnosis of immune suppression or collagen connective tissue disease, previous knee surgery, knee trauma or intraarticular injection in last six months, diagnosis of symptomatic hip or foot-ankle OA, presence of severe chronic illness, or poor general health status (heart failure, chronic bronchitis, etc.). In patients with bilateral symptoms, only the side with significant symptoms received treatment. The study protocol was approved by the Ethics Committee of Katip Çelebi University Faculty of Medicine Ethics Committee (Approval no: 57, approval date: 01.04.2015). A written informed consent was obtained from each patient. The study was conducted in accordance with the principles of the Declaration of Helsinki.

Patients were randomized by generating a random allocation sequence with a computer software program as corticosteroid group (receiving one corticosteroid injection), single PRP group (receiving one PRP injection), and three PRP group (receiving three PRP injections with one week interval) (Figure 1).

Peripheral venous blood (18 $\mathrm{mL}$ ) was collected with 18 gauge $(G)$ needle under aseptic conditions from the patients in the PRP groups to an injector containing $2 \mathrm{~mL}$ citrate dextrose. Collected blood was transferred to the kit. The PRP kit was 


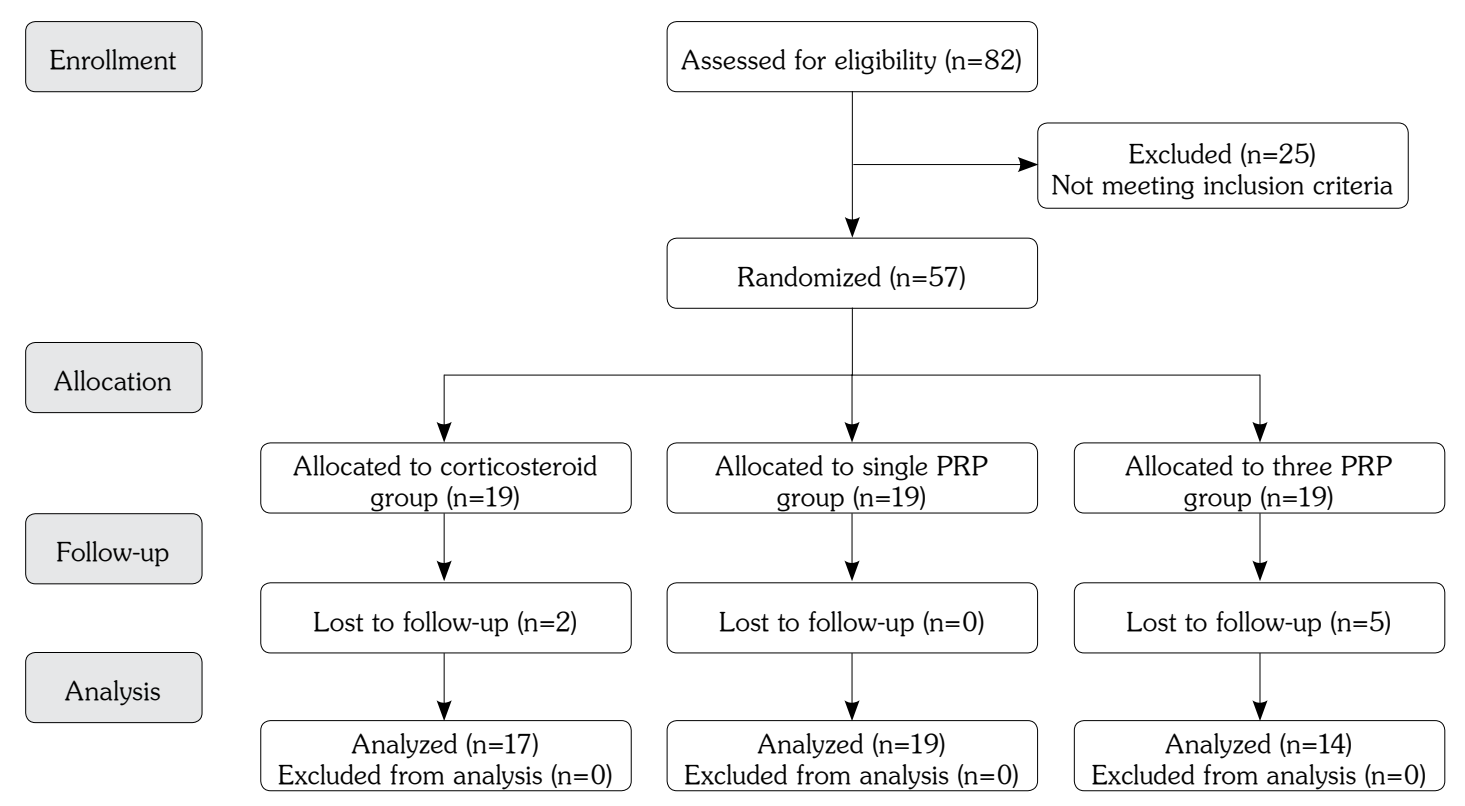

Figure 1. Study flow chart.

equilibrated with another kit of the same weight and centrifuged for five minutes at $3.600 \mathrm{rpm}$. Whole blood in the kit was separated as plasma on top, buffy coat containing platelets and leucocytes in the middle and as erythrocytes at the bottom.

Before the study, a platelet count was conducted with thrombocyte rich plasma sample, obtained from the same commercial kit in our biochemistry laboratory. PRP's platelet levels were compared with levels of the peripheral blood. The platelet count was $24510^{9} \mathrm{~L}$ and white blood cell (WBC) count was $7.4510^{9} \mathrm{~L}$ before centrifugation. After centrifugation, the platelet count was $87510^{9} \mathrm{~L}$ and WBC count was $8.6710^{9} \mathrm{~L}$.

The corticosteroid treatment group was treated once with a $1 \mathrm{~mL}$ suspension containing $6.43 \mathrm{mg}$ of betamethasone dipropionate (equivalent to $5.0 \mathrm{mg}$ of betamethasone) and $2.63 \mathrm{mg}$ of betamethasone sodium phosphate (equivalent to $2.0 \mathrm{mg}$ betamethasone).

All patients were treated with intraarticular injection with lateral-parapatellar approach. The same physician performed injections by using anatomical landmarks. The knee was immobilized for 10 minutes after the injection, and the patient was observed for an hour. All patients were recommended rest for 24-48 hours after discharge. In case of pain and swelling, superficial cold application for 10 minutes per hour was recommended. Patients were informed that their use of medications to prevent platelet aggregation during treatment might adversely affect treatment responses. When necessary, oral paracetamol was recommended (maximum $2 \mathrm{~g} /$ day). Before the treatment, all patients were taught quadricepsstrengthening exercises to perform 10 repeats per day in three sets. They were instructed to start a week after the application of treatment.

A trained and experienced physician blinded to the patients and the content of the injections evaluated the patients. Demographic data of the patients (age, sex, occupation, educational status, and Body Mass Index [BMI]), the side of the complaint and the duration of the complaint of knee pain were recorded. All patients were evaluated with Visual Numeric Scale (VNS) and Western Ontario and McMaster Universities Arthritis Osteoarthritis Index (WOMAC) pain scale, WOMAC stiffness scale for stiffness in the knee joint, WOMAC physical function scale and Lequesne scale for physical function. The anxiety and depression levels were evaluated by Hospital Anxiety and Depression (HAD) scale. These assessments were performed before the treatment, and at two-month and six-month follow-ups. 


\section{Statistical analysis}

The obtained data were analyzed using SPSS for Windows version 16.0 (SPSS Inc., Chicago, IL, USA) software. In the analysis of qualitative data, Chi-square test was used. The intra-group and inter-group parametric conditions were evaluated by Kolmogorov-Simirnov normality test. The time-varying scores of the groups were assessed with repeated measures analysis of variance test. The results were given as mean \pm standard error. Kruskal-Wallis analysis of variance and Mann-Whitney $U$ tests were used to investigate differences between groups. $\mathrm{P}<0.05$ values were considered statistically significant.

A priori required sample size was calculated using the G*Power version 3.1.9.2 software (Heinrich Heine University, Dusseldorf, Germany) based on the change in the pain score. The sample size was calculated using the combination of power (0.80), $\alpha$ (0.05), effect size (0.25), repetitive inter-measure correlation (0.5) and $\varepsilon$ (1.0) in accordance with the study design (bidirectional [time and treatment] analysis of variance in repeated measures). As a result, it was found that at least 12 patients (totally 36) in each group should be included in the study in order to reject the hypothesis of indifference.

\section{RESULTS}

Corticosteroid group consisted of 17 patients (2 males, 15 females; mean age 62.8 11.7 years, range 50 to 75 years), single PRP group consisted of 19 patients ( 1 male, 18 females; mean age
$62.3 \pm 1.6$ years; range 50 to 75 years) and three PRP group consisted of 14 patients (1 male, 13 females; mean age $60.4 \pm 1.7$ years; range

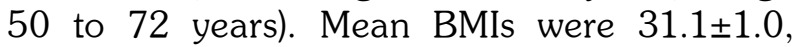
$31.4 \pm 0.7$, and $31.0 \pm 1.0$, respectively. There was no significant difference in age, weight and BMI between the groups ( $p>0.05)$.

When the pretreatment test scores of the patients were compared, three PRP group's WOMAC stiffness score was significantly higher than other treatment groups $(p<0.05$ with corticosteroid group and $\mathrm{p}<0.001$ with single PRP group).

In the corticosteroid group; VNS rest, night and movement scores were significantly decreased in the second month follow-up compared to baseline $(p<0.01)$. At sixth month, VNS rest and night scores were significantly higher than the second month $(p<0.05)$. Although there were no differences between baseline and sixth month VNS rest and night scores, the VNS movement scores at six month were still significantly lower than baseline $(p<0.01)$. There was no difference between the second and sixth month VNS movement scores. In single PRP group; VNS rest, night, and movement scores were significantly lower than baseline at second month $(p<0.01)$, and remained low up to sixth month follow-up $(p<0.01)$. There was no difference between the sixth month and second month VNS scores. In three PRP group; VNS rest, night, movement scores decreased significantly $(p<0.01)$ compared to baseline at second month, and VNS night and movement scores remained lower up to sixth month $(p<0.01)$. There was no difference between the sixth month and second

\begin{tabular}{|c|c|c|c|}
\hline \multirow[t]{2}{*}{ Visual Numeric Scale scores/time } & Basal & $2^{\text {nd }}$ month & $6^{\text {th }}$ month \\
\hline & Mean \pm SD & Mean \pm SD & Mean \pm SD \\
\hline \multicolumn{4}{|l|}{ Rest } \\
\hline Corticosteroid & $5.4 \pm 0.7$ & $1.9 \pm 0.7^{*}$ & $4.1 \pm 0.8 \dagger$ \\
\hline Single platelet rich plasma & $4.6 \pm 0.7$ & $1.1 \pm 0.6^{*}$ & $2.2 \pm 0.7^{*}$ \\
\hline Three platelet rich plasma & $4.1 \pm 1.0$ & $0.1 \pm 0.1^{*}$ & $1.9 \pm 0.8$ \\
\hline \multicolumn{4}{|l|}{ Night } \\
\hline Corticosteroid & $5.4 \pm 0.8$ & $1.7 \pm 0.6^{*}$ & $4.0 \pm 0.9 \dagger$ \\
\hline Single platelet rich plasma & $6.2 \pm 0.8$ & $1.7 \pm 0.6^{*}$ & $2.3 \pm 0.8^{*}$ \\
\hline Three platelet rich plasma & $5.4 \pm 1.1$ & $0.4 \pm 0.3^{*}$ & $1.8 \pm 0.8^{*}$ \\
\hline \multicolumn{4}{|l|}{ Movement } \\
\hline Corticosteroid & $8.4 \pm 0.3$ & $4.9 \pm 0.8^{*}$ & $5.7 \pm 0.9^{*}$ \\
\hline Single platelet rich plasma & $6.9 \pm 0.8$ & $3.2 \pm 0.7^{*}$ & $3.1 \pm 0.8^{*}$ \\
\hline Three platelet rich plasma & $8.0 \pm 0.5$ & $1.6 \pm 0.5^{*}$ & $2.5 \pm 0.9^{*}$ \\
\hline
\end{tabular}




\begin{tabular}{|c|c|c|c|}
\hline \multirow[t]{2}{*}{ WOMAC scores/time } & Basal & $2^{\text {nd }}$ month & $6^{\text {th }}$ month \\
\hline & $\overline{\text { Mean } \pm \mathrm{SD}}$ & $\overline{M e a n} \pm \mathrm{SD}$ & $\overline{\text { Mean } \pm S D}$ \\
\hline \multicolumn{4}{|l|}{ Pain } \\
\hline Corticosteroid & $12.8 \pm 0.8$ & $6.1 \pm 1.3^{*}$ & $9.8 \pm 1.5 \dagger キ$ \\
\hline Single platelet rich plasma & $11.5 \pm 0.9$ & $4.5 \pm 0.8^{*}$ & $5.0 \pm 1.1^{*}$ \\
\hline Three platelet rich plasma & $11.6 \pm 1.0$ & $2.6 \pm 0.8^{*}$ & $4.9 \pm 1.5^{*}$ \\
\hline \multicolumn{4}{|l|}{ Stiffness } \\
\hline Corticosteroid & $4.5 \pm 0.9$ & $1.5 \pm 0.5^{*}$ & $3.5 \pm 0.7 \neq$ \\
\hline Single platelet rich plasma & $4.3 \pm 0.4$ & $1.2 \pm 0.5^{*}$ & $1.8 \pm 0.5^{*}$ \\
\hline Three platelet rich plasma & $6.4 \pm 0.4$ & $2.0 \pm 0.6^{*}$ & $1.6 \pm 0.7^{*}$ \\
\hline \multicolumn{4}{|l|}{ Function } \\
\hline Corticosteroid & $43.4 \pm 2.3$ & $22.2 \pm 3.9^{*}$ & $27.4 \pm 3.8^{*}$ \\
\hline Single platelet rich plasma & $42.2 \pm 2.5$ & $16.7 \pm 3.0^{*}$ & $17.5 \pm 3.7^{*}$ \\
\hline Three platelet rich plasma & $44.9 \pm 3.2$ & $17.9 \pm 4.8^{*}$ & $14.3 \pm 4.1^{*}$ \\
\hline \multicolumn{4}{|l|}{ Total } \\
\hline Corticosteroid & $59.7 \pm 3.2$ & $29.7 \pm 5.3^{*}$ & $40.4 \pm 5.6^{*} \ddagger$ \\
\hline Single platelet rich plasma & $58.1 \pm 3.3$ & $22.4 \pm 4.1^{*}$ & $24.3 \pm 5.1^{*}$ \\
\hline Three platelet rich plasma & $62.9 \pm 4.2$ & $22.6 \pm 6.1^{*}$ & $24.6 \pm 7.1^{*}$ \\
\hline
\end{tabular}

month VNS scores. The sixth month VNS rest score did not show any difference compared to baseline and second month (Table 1).

In corticosteroid group; WOMAC pain, stiffness, function, and total scores were significantly lower at second month compared to baseline $(p<0.01)$. The sixth month WOMAC pain and total scores increased significantly compared to the second month $(p<0.05)$, but still remained lower than baseline $(p<0.05)$. The sixth month WOMAC function score was lower than baseline $(p<0.01)$ and no difference was found compared to the second month scores. The WOMAC stiffness score increased significantly at sixth month compared to second month $(p<0.05)$. In single and three PRP groups; WOMAC pain, stiffness, function, and total scores decreased significantly at second month compared to baseline $(p<0.01)$. The sixth month scores were also significantly low $(p<0.01)$. There was no

Table 3. Time-dependent changes in Lequesne scores of treatment groups

\begin{tabular}{|c|c|c|c|}
\hline \multirow[t]{2}{*}{ Lequesne scores/time } & Basal & $2^{\text {nd }}$ month & $6^{\text {th }}$ month \\
\hline & $\overline{\text { Mean } \pm \text { SD }}$ & $\overline{\text { Mean } \pm S D}$ & $\overline{\text { Mean } \pm S D}$ \\
\hline Corticosteroid & $13.6 \pm 0.7$ & $8.0 \pm 1.4^{*}$ & $10.9 \pm 1.1 \dagger \neq$ \\
\hline Single platelet rich plasma & $12.4 \pm 1.0$ & $7.1 \pm 1.1^{*}$ & $8.0 \pm 1.4^{*}$ \\
\hline Three platelet rich plasma & $14.5 \pm 1.0$ & $6.6 \pm 1.0^{*}$ & $7.8 \pm 1.3^{*}$ \\
\hline
\end{tabular}

Table 4. Differences between treatment groups

\begin{tabular}{|c|c|c|c|}
\hline \multirow[t]{2}{*}{ Lequesne scores/time } & $\begin{array}{c}2^{\text {nd }} \text { month } \\
\text { VNS (movement) }\end{array}$ & $\begin{array}{c}6^{\text {th }} \text { month } \\
\text { VNS (movement) }\end{array}$ & $\begin{array}{c}6^{\text {th }} \text { month } \\
\text { WOMAC (pain) }\end{array}$ \\
\hline & Mean \pm SD & Mean \pm SD & Mean \pm SD \\
\hline Corticosteroid & $4.9 \pm 0.8^{*}$ & $5.7 \pm 0.9+キ$ & $9.8 \pm 1.5 \dagger \neq$ \\
\hline Single platelet rich plasma & $3.2 \pm 0.7$ & $3.1 \pm 0.8 \dagger$ & $5.0 \pm 1.1 \dagger$ \\
\hline Three platelet rich plasma & $1.6 \pm 0.5^{*}$ & $2.5 \pm 0.9 \neq$ & $4.9 \pm 1.5 \neq$ \\
\hline
\end{tabular}



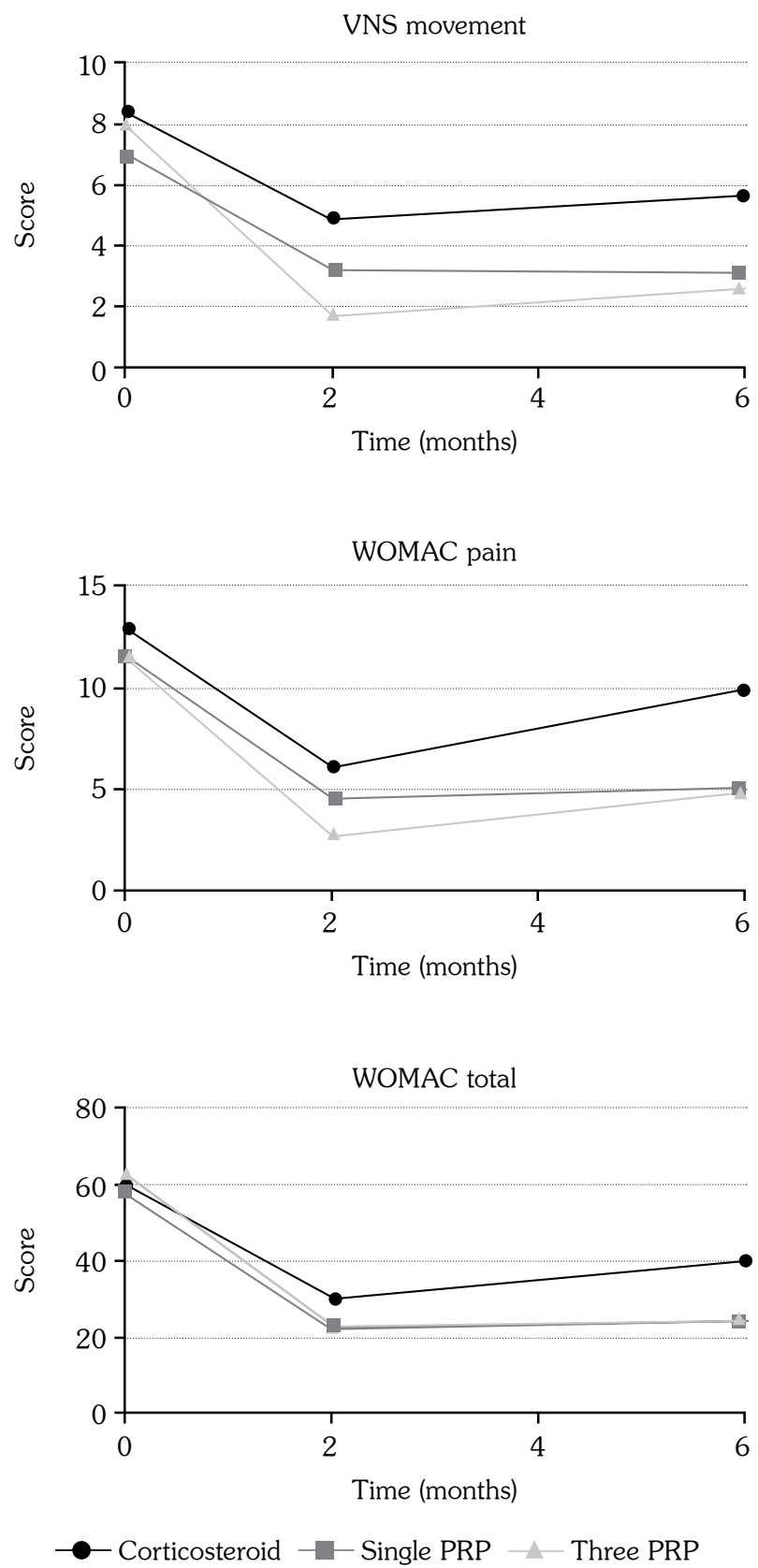

Figure 2. Time-dependent changes in Visual Numeric Scale (movement), and Western Ontario and McMaster Universities Arthritis Osteoarthritis Index pain and total scores. VNS: Visual Numeric Scale; WOMAC: Western Ontario and McMaster Universities Arthritis Osteoarthritis Index; PRP: Platelet rich plasma.

difference between the sixth month and second month scores (Table 2).

In corticosteroid group, Lequesne scores of second month were significantly lower than baseline $(p<0.01)$. The sixth month scores increased significantly compared to the second month scores $(p<0.05)$; but remained significantly lower than baseline score $(p<0.05)$. In single and three PRP groups, Lequesne scores were significantly lower than baseline at second and sixth month follow-ups $(p<0.01)$, with no significant difference between the second month and sixth month scores (Table 3).

In corticosteroid group; basal HAD anxiety and depression scores were $8.2 \pm 1.1$ and $4.88 \pm 0.86$; in single PRP group $8.8 \pm 1.2$ and $4.8 \pm 0.9$; and in three PRP group $11.8 \pm 1.4$ and $7.3 \pm 1.5$, respectively. There was no change in HAD anxiety and depression scores in the corticosteroid group. In the single PRP group, the HAD anxiety scores were decreased in the second and sixth month follow-ups compared to baseline $(p<0.01)$. In the three PRP group, HAD anxiety and depression scores were significantly lower at sixth month compared to baseline and second month follow-up $(\mathrm{p}<0.05$ and $\mathrm{p}<0.01)$.

When the groups were compared among themselves, three PRP injection group's VNS movement scores at second month were significantly lower than corticosteroid group $(p<0.01)$. The sixth month VNS movement scores of the three PRP and single PRP groups were also significantly lower than the corticosteroid group $(\mathrm{p}<0.05)$. The sixth month WOMAC pain scores of the three PRP and single PRP groups were significantly lower when compared to the corticosteroid group $(p<0.05)$. There was no difference between PRP groups in terms of VNS movement and WOMAC pain scores at sixth month (Table 4) (Figure 2).

\section{DISCUSSION}

There have been many studies investigating the effectiveness of PRP in degenerative knee disease. In their study published in 2009, Kon et al. $^{10}$ found significant improvement in pain scores of patients who received three PRP injections with three-week intervals at second and sixth month follow-ups. The results were stable until sixth month, while scores worsened at $12^{\text {th }}$ month follow-up. In the $24^{\text {th }}$ month follow-up of the same patients, pain scores were still better than baseline, but had worsened compared to $12^{\text {th }}$ month controls. The mean duration of 
clinical wellness was nine months. Better clinical response was obtained in younger patients with early stage cartilage lesions. ${ }^{7}$ In a multi-center prospective study published in 2011 by Kon et al., ${ }^{10}$ PRP treatment was compared with high and low molecular weight hyaluronic acid injections and PRP was found superior in terms of efficacy at sixth month control. In the subgroup analysis of this study, chondropathy group had responded better to the treatment than early stage and late stage OA group. Also, patients younger than 50 years had benefited better from growth factor therapy. ${ }^{11}$ Görmeli et al. ${ }^{12}$ compared three injections of PRP with one week interval and single injections of PRP, hyaluronic acid and saline (placebo) and demonstrated that all groups except for placebo improved significantly. Compared with the other treatment groups, knee scores were better in the group with three PRP injections and there was no difference between single dose PRP and hyaluronic acid injections. In the early stage OA subgroups, a better clinical outcome was obtained in three PRP treatment groups; however, there was no difference between treatment outcomes in late stage OA groups.

In a systematic review published in 2015 by Meheux et al., ${ }^{13}$ intraarticular PRP therapy was found superior to the intraarticular hyaluronic acid therapy in improving WOMAC scores and maintaining clinical wellness at three to 12 months after injection. In the same study, PRP injections were reported to provide better clinical improvement in younger and physically active patients with low grade cartilage damage. In a meta-analysis by Laudy et al. ${ }^{14}$ in 2015, PRP injections were found to be more effective than placebo in reducing pain at sixth month after injection. Compared with hyaluronic acid, PRP was more effective in reducing pain at sixth month according to VAS and NRS scales. Physical function was also significantly improved with PRP treatment. In our study; in PRP groups; VNS, WOMAC, and Lequesne scores were significantly decreased at second month follow-up. Although there was a slight increase at sixth month, they were still low compared to baseline.

The results of these randomized controlled trials and meta-analyses are promising; nevertheless, the ideal PRP preparation method and application schedule have not yet been determined. ${ }^{8,15-17}$ Meheux et al. ${ }^{13}$ have concluded that PRP injections could be administered in two-four sessions at two-four weeks apart to patients with symptomatic knee OA between Kellgren-Lawrence grades 1 to 3 . In their systemic review and meta-analysis published in 2014, Chang et al. ${ }^{18}$ reported that two or fewer injections may reduce the effectiveness of treatment and a minimum of three injections were recommended. In our study, there was no difference between the single application and three consecutive application groups. This may be due to the fact that in our study, the intervals between injections were shorter than those in the meta-analyses. It may also be due to late stage cartilage damage in our study patients.

Platelet rich plasma has been reported as a safe treatment with no serious complications. Minor side effects reported with repeated intraarticular injections are pain, swelling and mild effusion that can last a few days. ${ }^{16}$ In our study, we only observed a mild erythema on one patient's knee in the single PRP group, which regressed with cold application in six hours. We have not experienced any complaints of local swelling or pain.

Intraarticular corticosteroid injections are widely used to reduce pain and limitation of joint movement in knee OA, particularly in the presence of inflammation and joint effusion. ${ }^{16}$ In the Cochrane review at 2006, intraarticular corticosteroid injections were found effective in reducing pain up to three weeks when compared with placebo in knee OA, but not in terms of functional improvement. There was no significant improvement in pain and function at 4 to 24 weeks after injection. When compared with hyaluronic acid injections, there was no difference in the first four weeks after injection. However, hyaluronic acid was found to be superior in terms of WOMAC OA scores, Lequesne index, pain control, and joint range of motion improvement between fifth and $13^{\text {th }}$ weeks. Its efficacy was found similar to corticosteroids but then, more durable. ${ }^{19}$ In our study; all VNS, WOMAC and Lequesne scores decreased significantly in the second month follow-up in patients who were injected with intraarticular corticosteroids. However, VNS movement and WOMAC stiffness scores increased at the end of the sixth month, and no difference was detected compared to baseline 
scores. Sixth month VNS movement, WOMAC pain, function, total and Lequesne scores were worse compared to the second month, but were significantly lower than before the treatment.

In a randomized controlled trial conducted by Gobbi et al., ${ }^{9}$ intraarticular single dose PRP and corticosteroids were compared in patients with grade 2-3 OA. Pain, symptoms, activities of daily living and quality of life were significantly improved in the PRP group compared to the corticosteroid group at second and sixth month follow-ups. In our study, there was no difference between the groups in terms of WOMAC and Lequesne scores at second month. However, three PRP group's VNS movement score at second month was significantly lower than that of the corticosteroid group. In the sixth month follow-up, VNS movement and WOMAC pain scores of the three PRP and single PRP groups were also significantly lower than the corticosteroid group. Another difference emerged in evaluating HAD scores. When the HAD anxiety and depression scores were evaluated in the corticosteroid group, there was no significant difference between the baseline, second and sixth month scores, whereas the HAD anxiety score in the group with single PRP injection decreased significantly in second and sixth month follow-ups. In the patient group with three consecutive PRP injections, HAD anxiety and depression scores were significantly lower in the sixth month than baseline and second month follow-up.

Our study has several limitations. First of all, our sample size was small and follow-up period was short. Due to the fact that the numbers of injections were different and placebo was not used, patient groups were not blinded. Another limitation is that injections were not performed under ultrasound guidance.

In conclusion, our findings have shown that intraarticular PRP injections are safe and effective treatment options up to six months in OA symptom control. Treatment response with corticosteroid therapy is shorter when compared to PRP injection therapy. There was no significant difference between single and consecutive injections of PRP injections. More studies are required in this area to determine a standard PRP preparation method and application period and to assess long-term treatment efficacy.

\section{Declaration of conflicting interests}

The authors declared no conflicts of interest with respect to the authorship and/or publication of this article.

\section{Funding}

The authors received no financial support for the research and/or authorship of this article.

\section{REFERENCES}

1. Şavluk ÖF, Baysal A, Erbaş M, Toman H, Daldal E. Dejeneratif diz osteoartriti (OA) olan hastalarda intraartiküler steroid uygulamasının etkinliği. Düzce Tip Dergisi 2013;15:27-31.

2. Michael JW, Schlüter-Brust KU, Eysel P. The epidemiology, etiology, diagnosis, and treatment of osteoarthritis of the knee. Dtsch Arztebl Int 2010;107:152-62.

3. Hochberg MC, Altman RD, April KT, Benkhalti M, Guyatt G, McGowan J, et al. American College of Rheumatology 2012 recommendations for the use of nonpharmacologic and pharmacologic therapies in osteoarthritis of the hand, hip, and knee. Arthritis Care Res (Hoboken) 2012;64:465-74.

4. McAlindon TE, Bannuru RR, Sullivan MC, Arden NK, Berenbaum F, Bierma-Zeinstra SM, et al. OARSI guidelines for the non-surgical management of knee osteoarthritis. Osteoarthritis Cartilage 2014;22:363-88.

5. Bannuru RR, Natov NS, Obadan IE, Price LL, Schmid $\mathrm{CH}$, McAlindon TE. Therapeutic trajectory of hyaluronic acid versus corticosteroids in the treatment of knee osteoarthritis: a systematic review and metaanalysis. Arthritis Rheum 2009;61:1704-11.

6. Rutjes $\mathrm{AW}$, Jüni $\mathrm{P}$, da Costa BR, Trelle $\mathrm{S}$, Nüesch E, Reichenbach S. Viscosupplementation for osteoarthritis of the knee: a systematic review and meta-analysis. Ann Intern Med 2012;157:180-91.

7. Filardo G, Kon E, Buda R, Timoncini A, Di Martino A, Cenacchi $A$, et al. Platelet-rich plasma intra-articular knee injections for the treatment of degenerative cartilage lesions and osteoarthritis. Knee Surg Sports Traumatol Arthrosc 2011;19:528-35.

8. Gobbi A, Karnatzikos G, Mahajan V, Malchira S. Platelet-rich plasma treatment in symptomatic patients with knee osteoarthritis: preliminary results in a group of active patients. Sports Health 2012;4:162-72.

9. Forogh B, Mianehsaz E, Shoaee S, Ahadi T, Raissi GR, Sajadi S. Effect of single injection of platelet-rich plasma in comparison with corticosteroid on knee osteoarthritis: a double-blind randomized clinical trial. J Sports Med Phys Fitness 2016;56:901-8.

10. Kon E, Buda R, Filardo G, Di Martino A, Timoncini A, Cenacchi A, et al. Platelet-rich plasma: intraarticular knee injections produced favorable results on degenerative cartilage lesions. Knee Surg Sports Traumatol Arthrosc 2010;18:472-9. 
11. Kon E, Mandelbaum B, Buda R, Filardo G, Delcogliano M, Timoncini A, et al. Platelet-rich plasma intra-articular injection versus hyaluronic acid viscosupplementation as treatments for cartilage pathology: from early degeneration to osteoarthritis. Arthroscopy 2011;27:1490-501.

12. Görmeli G, Görmeli CA, Ataoglu B, Çolak C, Aslantürk O, Ertem K. Multiple PRP injections are more effective than single injections and hyaluronic acid in knees with early osteoarthritis: a randomized, double-blind, placebo-controlled trial. Knee Surg Sports Traumatol Arthrosc 2017;25:958-65.

13. Meheux CJ, McCulloch PC, Lintner DM, Varner KE, Harris JD. Efficacy of Intra-articular PlateletRich Plasma Injections in Knee Osteoarthritis: A Systematic Review. Arthroscopy 2016;32:495-505.

14. Laudy AB, Bakker EW, Rekers M, Moen MH. Efficacy of platelet-rich plasma injections in osteoarthritis of the knee: a systematic review and meta-analysis. Br J Sports Med 2015;49:657-72.

15. Riboh JC, Saltzman BM, Yanke AB, Fortier L, Cole BJ. Effect of Leukocyte Concentration on the Efficacy of Platelet-Rich Plasma in the Treatment of Knee Osteoarthritis. Am J Sports Med 2016;44:792-800.

16. Ayhan E, Kesmezacar H, Akgun I. Intraarticular injections (corticosteroid, hyaluronic acid, platelet rich plasma) for the knee osteoarthritis. World J Orthop 2014;5:351-61.

17. Filardo G, Kon E, Pereira Ruiz MT, Vaccaro F, Guitaldi R, Di Martino A, et al. Platelet-rich plasma intra-articular injections for cartilage degeneration and osteoarthritis: single- versus double-spinning approach. Knee Surg Sports Traumatol Arthrosc 2012;20:2082-91.

18. Chang KV, Hung CY, Aliwarga F, Wang TG, Han DS, Chen WS. Comparative effectiveness of platelet-rich plasma injections for treating knee joint cartilage degenerative pathology: a systematic review and meta-analysis. Arch Phys Med Rehabil 2014;95:562-75.

19. Bellamy N, Campbell J, Robinson V, Gee T, Bourne R, Wells G. Intraarticular corticosteroid for treatment of osteoarthritis of the knee. Cochrane Database Syst Rev 2006;2:CD005328. 